\title{
Developmental changes of the contingent negative variation in migraine and healthy children
}

\author{
Michael Siniatchkin · Anne Jonas · Huelya Baki • \\ Andreas van Baalen · Wolf-Dieter Gerber • \\ Ulrich Stephani
}

Received: 17 August 2009/Accepted: 24 November 2009/Published online: 15 December 2009

(C) Springer-Verlag 2009

\begin{abstract}
It has been hypothesized that abnormalities of information processing in migraine may be attributed to impairment of cerebral maturation. However, the most evidences for this hypothesis have come from cross-sectional studies during childhood. We performed a longitudinal study and recorded contingent negative variation $(\mathrm{CNV})$, an event-related slow cortical potential, in migraine children $(n=27)$ and age-matched healthy individuals $(n=23)$ in 1998 and 8 years later (2006). Amplitudes of all $\mathrm{CNV}$ components were reduced and habituation of the initial $\mathrm{CNV}$ ( $\mathrm{iCNV}$ ) increased in the observed time. However, the reduction of the iCNV amplitude was more pronounced in migraine patients who were in remission in 2006 and in healthy subjects and less pronounced in migraineurs with persisting headaches. Patients with the worsened migraine demonstrated the most pronounced loss of iCNV habituation in 1998 and significantly increased iCNV amplitudes in 2006. This longitudinal study supports the hypothesis of impaired cerebral maturation in migraine and shows that migraine manifestation is a key factor interfering with the natural maturation process of central information processing.
\end{abstract}

Keywords Migraine - Contingent negative variation . Habituation · Maturation

M. Siniatchkin $(\bowtie) \cdot$ A. Jonas · H. Baki · A. van Baalen ·

U. Stephani

Department of Child Neurology, Christian-Albrechts-University

of Kiel, Schwanenweg 20, 24105 Kiel, Germany

e-mail: m.siniatchkin@pedneuro.uni-kiel.de

W.-D. Gerber

Institute of Medical Psychology, Christian-Albrechts-University

of Kiel, Kiel, Germany

\author{
Abbreviations \\ CNV Contingent negative variation \\ iCNV Initial CNV \\ ICNV Late CNV \\ PINV Post-imperative negative variation \\ RT Reaction time \\ TTH Tension-type headache
}

\section{Introduction}

According to current concepts of migraine pathogenesis, abnormal cortical excitability and altered cortical information processing seem to represent fundamental features of the brains of migraineurs [1]. In the last two decades, the contingent negative variation (CNV) has been established as an appropriate method to investigate both these features [2]. CNV is a slow cortical, event-related potential which can be recorded from the scalp between two stimuli (warning [S1], and imperative stimulus [S2]) during expectancy [3]. From a physiological point of view, the CNV amplitude reflects the excitability of the dendritic trees of cortical pyramidal neurons following activation in the cortico-striato-thalamo-cortical loop [4]. If longer (>3 s) inter-stimulus intervals are employed, sub-components of the CNV can be separated. The initial CNV component (iCNV, 0.5-1.0 s after S1) has been related to the orienting response [5, 6]. The late CNV component ( $\mathrm{CNNV}$, some hundred ms before S2) is believed to represent the stimulus anticipation and response preparation [7, 8].

Migraine patients are characterized by increased amplitude as well as reduced habituation of the CNV [9]. Especially, the amplitude of the iCNV component and its habituation are abnormal in migraine [10-16]. In 1999, 
Kropp et al. described differences in iCNV between adult patients suffering from migraine without aura and healthy subjects but not children or adolescents. In the ages 15-19 years, healthy adolescents demonstrated a significant reduction of the $\mathrm{iCNV}$ amplitude and a substantial increase of the iCNV habituation. However, these developmental changes did not occur in migraineurs [17]. It was proposed that the described differences between migraine patients and healthy subjects in iCNV amplitude and habituation found in adults might be due to an impaired maturation of sensory information processing in migraine patients $[13,17]$. The concept of impaired sensory maturation in migraine was supported by other studies which used measurement of the intensity dependence of auditory evoked potentials $[18,19]$ and habituation of visual evoked potentials [20, 21]. Although recent CNV studies encouraged the concept too, they demonstrated a lack of agedependent development of the 1CNV but not of the iCNV $[22,23]$. Among methodological differences, the contradictions between the studies may have been caused by one important bias: most of the previous studies employed a cross-sectional design. Thus, there is a crucial need for longitudinal studies in order to prove the hypotheses of migraine being a disorder of impaired sensory maturation. The advantage of longitudinal studies was shown by Oelker-Ax et al. [24]. The authors demonstrated lack of development for late and total $\mathrm{CNV}$ in children with migraine after 4 years follow-up.

The aim of this study was to investigate changes of the CNV over 8 years in children and adolescents suffering from migraine without aura as well as in healthy, agematched subjects using a longitudinal study design. The changes of CNV were correlated with the clinical course of migraine during this time.

\section{Patients and method}

\section{Participants}

A two-point longitudinal assessment was done in 19971998 (T1, $n=93$ participants) and 2006-2007 (T2, $n=50$ participants). At T1, 45 patients suffering from migraine without aura (age $10.37 \pm 2.45$ years, 28 boys) and 48 headache-free control children and adolescents without family history of migraine (age $11.33 \pm 2.66$ years, 21 boys) were investigated. At T2, 27 (60\%) migraine children and $23(48 \%)$ healthy controls could be followed up. The main reason for healthy subjects dropping out was noncompliance (lack of motivation to take part in the study once again). The reason for migraine patients dropping out was either loss of contact or non-compliance in a few children. There were no differences between dropouts and participants according to clinical course of headache, CNV characteristics, and psychopathological parameters at T1. Migraine and healthy families were recruited from the outpatient department of the University Hospital of Paediatric Neurology, Kiel. Healthy children were recruited from local schools. For details regarding the recruitment procedure, diagnostic arrangements, demographic and clinical characteristics of subjects at $\mathrm{T} 1$ see [13].

At T2, structured headache interviews were performed with all subjects and the migraine diagnosis was re-evaluated according to the revised criteria of the International Headache Society [25]. All diagnoses of migraine at T1 were supported using revised diagnostic criteria. In cases of failed remission, all patients with migraine without aura at T1 (IHS code 1.1.) still had the same type of headache at T2. Subjects reporting both migraine and tension-type headache (TTH) were included into the migraine group. Some patients developed very frequent headaches (see the group with worsened migraine) which did not fulfill diagnostic criteria for chronic migraine or chronic TTH. However, chronic daily headache cannot be excluded because of a short observation period. None of the patients demonstrated a transformation of migraine into the TTH. Additionally, a child-adapted prospective daily headache diary was used to assess headache characteristics over a four-week-period prior to the investigation [26]. The diagnosis of migraine was validated by the headache diary in all patients without remission. Both the diary data (absence of migraine) and the history (no migraine headaches in the previous 6 months) were taken as criteria for remission. Both criteria were fulfilled in all cases.

Demographic and clinical characteristics of participants at T2 are presented in Table 1. Neurological and internal medical examinations revealed no health problems (including acute infection) other than primary headaches in all participants. None of the subjects presented with any psychiatric disorder, which would have fulfilled the diagnostic criteria of DSM-IV-TR (4th Edition, American Psychiatric Association, Washington, DC, 2000). None of the participants used any medication or took part in any non-pharmacological treatment programs for at least 6 months prior to the investigation. None of the patients took acute medication more than two times a month. Care was taken that the time interval between the attacks and the recording be at least 5 days as $\mathrm{CNV}$ amplitudes show periodic changes in the migraine interval [11, 12]. The influence of the current state of the participants on the CNV was considered by recording the quality and duration of sleep during the night before investigation, assessing mood (subjective evaluation on the digital scale from 1 to 7 ), present medications, as well as drug, alcohol and caffeine intake on the day of recording using a standardized questionnaire. There were no differences between the groups 
Table 1 Demographic and clinical characteristics of participants
Mean age and duration of disease are given for $\mathrm{T} 2$.

The parameters are given as a mean \pm standard deviation

$T 1$ investigation in 1998, T2 investigation in 2006

\begin{tabular}{|c|c|c|c|c|}
\hline & \multirow[t]{2}{*}{ Healthy subjects } & \multicolumn{3}{|l|}{ Migraine patients } \\
\hline & & With remission & Improved & Worsened \\
\hline No. & 23 & 9 & 12 & 6 \\
\hline Men:women & $10: 13$ & $4: 5$ & $9: 3$ & $4: 2$ \\
\hline Age (years) & $18.1 \pm 1.9$ & $18.8 \pm 2.7$ & $19.1 \pm 2.8$ & $18.0 \pm 2.7$ \\
\hline Duration of disease (years) & & $13.4 \pm 1.9$ & $13.3 \pm 2.8$ & $13.2 \pm 1.3$ \\
\hline \multicolumn{5}{|l|}{ Days with migraine/months } \\
\hline $\mathrm{T} 1$ & & $2.4 \pm 2.8$ & $3.6 \pm 2.3$ & $2.2 \pm 1.3$ \\
\hline $\mathrm{T} 2$ & & - & $2.2 \pm 2.2$ & $7.2 \pm 6.2$ \\
\hline \multicolumn{5}{|l|}{ Duration of attack (h) } \\
\hline $\mathrm{T} 1$ & & $7.1 \pm 3.1$ & $7.1 \pm 3.6$ & $4.2 \pm 0.6$ \\
\hline $\mathrm{T} 2$ & & - & $4.8 \pm 5.2$ & $5.6 \pm 2.5$ \\
\hline \multicolumn{5}{|c|}{ Severity of attack from 1 to 5} \\
\hline $\mathrm{T} 1$ & & $4.7 \pm 0.7$ & $4.1 \pm 0.7$ & $4.2 \pm 0.8$ \\
\hline $\mathrm{T} 2$ & & - & $2.7 \pm 2.4$ & $5.1 \pm 0.6$ \\
\hline
\end{tabular}

with regard to any of these factors (one-way ANOVA and $\chi^{2}$ : non-significant). None of the subjects had a hearing impairment or had drunk alcohol during the 3 days before investigation. In girls, no recordings were performed in the premenstrual period of the cycle. Most of the recordings were carried out in the afternoon.

The study was permitted by the Ethic Committee of the Faculty of Medicine, University of Kiel, Germany. All participants and their parents were instructed about the study and written informed consent according to the Declaration of Helsinki (current version, 1996) on biomedical research involving human subjects (Tokyo amendment) was obtained. All subjects received money as a compensation for the efforts to participate and were highly motivated.

\section{Experimental design and recordings}

$\mathrm{CNV}$ was recorded at $\mathrm{T} 2$ using the same procedure and same equipment as at T1 [13]. All participants were seated in an armchair with eyes open in an electrically shielded soundproof room. The subjects were asked not to close or move their eyes or to blink. The auditory warning (S1) and imperative stimuli (S2) with an intensity of $75 \mathrm{~dB}$ were produced by a loudspeaker located behind the subject. The interval between $\mathrm{S} 1$ and $\mathrm{S} 2$ was 3 s. A CNV session consisted of 32 trials in which the subject was to react immediately to the imperative stimulus (GO-response). In addition, eight trials were randomly presented in which no reaction was expected (NO-GO-response). The warning stimulus (S1) for the GO-response had a frequency of $f=1,000 \mathrm{~Hz}$ and lasted $100 \mathrm{~ms}$. The warning tone for the NO-GO-response had a frequency of $f=200 \mathrm{~Hz}$. The imperative stimulus (S2) had a frequency of $f=2,500 \mathrm{~Hz}$, lasted a maximum of $1,500 \mathrm{~ms}$ and was deactivated by pressing the button with the dominant right hand. Reaction time was defined as the period between the onset of S2 and the pressing of the button. S1 and S2 pairs were offered at random intervals of $10-15 \mathrm{~s}$. The duration of the recorded epoch was $6 \mathrm{~s}$ (the recording began $1 \mathrm{~s}$ before $\mathrm{S} 1$ and ended $2 \mathrm{~s}$ after $\mathrm{S} 2$ ). The period between recording onset and $\mathrm{S} 1$ was taken as the baseline for all measurements.

The EEG was recorded using non-polarisable $\mathrm{Ag} / \mathrm{AgCl}$ electrodes over $\mathrm{Cz}$ according to the International 10-20 System with linked mastoids as reference. The electrode site on the scalp was prepared with alcohol and scraped with rough paper resulting in an electrode impedance of less than $10 \mathrm{k} \Omega$. There were no differences in electrode impedances between all investigated groups. The EEG signals were amplified using a Nihon Kohden amplifier with a time constant of $5 \mathrm{~s}$ (equivalent to a high-pass filter of $0.03 \mathrm{~Hz}$ ), a low-pass filter of $35 \mathrm{~Hz}$ and digitized at a rate of $100 \mathrm{~Hz}$ for each channel. Vertical eye movement artefacts were excluded by parallel recording of the electrooculogram (EOG) using electrodes $(\mathrm{Ag} / \mathrm{AgCl})$ positioned $5 \mathrm{~cm}$ above and below the right eye. The trial was rejected if EOG deflections greater than $20 \mu \mathrm{V}$ interfered with $5 \mathrm{~s}$ of the EEG recording. A protocol listed the number of rejected trials for each recording. There were no significant differences between the groups with respect to the number of rejected trials.

Data analysis

The GO-trials were averaged and the amplitudes of the total CNV, the iCNV and 1CNV components as well as the post-imperative negative variation (PINV) were calculated. The total CNV was assessed between 500 and 3,000 ms 
following S1. The iCNV was defined as the mean amplitude in a window of $200 \mathrm{~ms}$ around the maximal amplitude of the expectancy wave between 550 and $750 \mathrm{~ms}$ after S1 [27]. The ICNV was the mean amplitude during the $200 \mathrm{~ms}$ preceding S2. PINV was the mean amplitude of CNV between 500 and 2,000 ms following S2. Each recording was divided into eight blocks of four consecutive trials to determine the course of habituation and trends in the early $\mathrm{CNV}$ amplitudes. Habituation was indicated by a negative, whereas loss of habituation was marked by a positive slope as calculated by linear regression $(y=a x+b$, where $a$ is the slope of habituation and $b$ the intercept of linear regression) [28]. This study considered only the early CNV component of habituation data. This limitation of data is based on the literature and on observations made in the same group of patients as well as on the results at T1, whereby only the early CNV showed habituation differences between the groups [13].

\section{Statistical evaluation}

Since the data were normally distributed $(P>0.8$ in the Kolmogorov-Smirnov test) and characterized by homogeneous variances $(F$ test), parametric tests were applied. For clinical data, two-tailed $t$ statistics was calculated in groups of migraine patients. For CNV characteristics, main withinsubjects effect "Follow up" (T1 vs. T2) and betweensubjects effect "Group" (migraine patients with remission vs. patients with improved migraine vs. patients with worsened migraine vs. Healthy subjects) and the interaction "Follow-up $\times$ Group" were determined using MANOVA. The comparisons between the groups at $\mathrm{T} 1$ and T2 were performed with one-way ANOVA. Any observed effects in the between-subjects were evaluated with post hoc Bonferoni tests and corrected for multiple comparisons $(P<0.05$ corrected). The comparisons between $\mathrm{T} 1$ and $\mathrm{T} 2$ for CNV parameters were performed using paired-samples $t$ tests and subsequent Bonferroni alpha adjustment. All statistical evaluations were made using SPSS for Windows 11.0 (SPSS Inc., Chicago, USA).

\section{Results}

\section{Clinical course of migraine}

Table 1 represents demographic and clinical characteristics of migraine at T1 (1998) and T2 (2006) in each group. In the migraine group, $9(33 \%)$ patients became headachefree, 18 (67\%) patients still reported migraine without aura. In these patients, migrainous headaches were also documented in a headache diary. Headache frequency was taken as a criterion for improvement or worsening of headaches.
In most patients with enduring migraine, the headaches improved in 12 patients (reduced frequency of migraine in a headache diary in 2006 compared with 1998). In the other six patients, the migrainous headache worsened over time (increased frequency of migraine). The comparison of headache characteristics at $\mathrm{T} 1$ between patients with improved and worsened migraine and subjects who became headache-free revealed no significant differences. Two children from the group of healthy subjects developed migrainous headaches and were therefore excluded from further analysis. The CNV parameters in these children did not differ from those in the group of migraine patients with worsened headaches. However, larger group of newly diagnosed subjects with migraine is needed to make conclusions about the value of $\mathrm{CNV}$ as a predictor for development of migraine over time.

\section{Changes in $\mathrm{CNV}$ characteristics}

Amplitudes of all CNV components decrease over time in healthy subjects, migraineurs with persisting headaches and migraineurs with remission (Fig. 1). The same is true for iCNV habituation which increases in all groups during the follow-up (Fig. 2). The reaction time seems to decrease with age: the older the subjects, the faster the reaction time.

Statistical analysis corroborates these statements (Table 2). The main effect "Follow-up" was significant for all components, habituation coefficient as well as for the reaction time. This indicates that all $\mathrm{CNV}$ parameters changed between T1 and T2. The main effect "Group" was significant for the coefficient of CNV habituation and for the iCNV component. This suggests that iCNV amplitude and habituation differ between the groups either at $\mathrm{T} 1$ or at T2. The interaction "Follow $\times$ Group" was significant for both iCNV amplitude and coefficient of CNV habituation. This means that the $\mathrm{iCNV}$ and its habituation undergo different changes over time in the investigated groups.

The subsequent ANOVAs (comparisons between groups separately for T1 and T2) demonstrated that the described difference in the iCNV habituation occurred at T1 $[F(3$, $44)=3.37 ; P=0.03]$ and the difference in the iCNV amplitude can be attributed to both $\mathrm{T} 1[F(3,44)=4.32$; $P=0.009]$ and T2 $[F(3,44)=4.02 ; P=0.01]$. No other significant differences between groups, neither for T1 nor for T2, were found. Post hoc tests revealed significantly higher coefficients of habituation in the group of migraine patients with worsened headache compared with migraineurs with improved migraine $(P=0.04$, corrected $)$ or healthy control subjects $(P=0.02$, corrected). Indeed, patients with enduring headaches who worsened in the 8-year follow-up demonstrated pronounced loss of habituation in 1998. At T1, the iCNV amplitude was significantly increased in the group of migraine patients who 
Fig. 1 Amplitudes (mean and standard deviation) of different CNV components which were determined at T1 (1998) and T2 (2006) in healthy subjects and migraine patients (with remission, improved or worsened headaches). Reaction time (mean and standard deviations in parentheses) is given in the figure with PINV. $P$ values demonstrate difference (paired $t$ tests) between amplitudes of the iCNV obtained at T1 and T2. These differences did not survive Bonferroni alpha adjustment for the late CNV component and the total CNV

Fig. 2 Course of habituation of the iCNV determined at $\mathrm{T} 1$ (1998) and T2 (2006) in healthy subjects and migraine patients (with remission, improved or worsened headaches).

Amplitudes in eight blocks of recordings are given as mean and standard error. Regression lines are supported by equations $y=\boldsymbol{A}(\boldsymbol{z}) x+\boldsymbol{B}(\boldsymbol{u p})$ where $\boldsymbol{A}$ is a mean of regression coefficient and $z$ its standard deviation; $\boldsymbol{B}$ an intercept and $u p$ its standard deviation
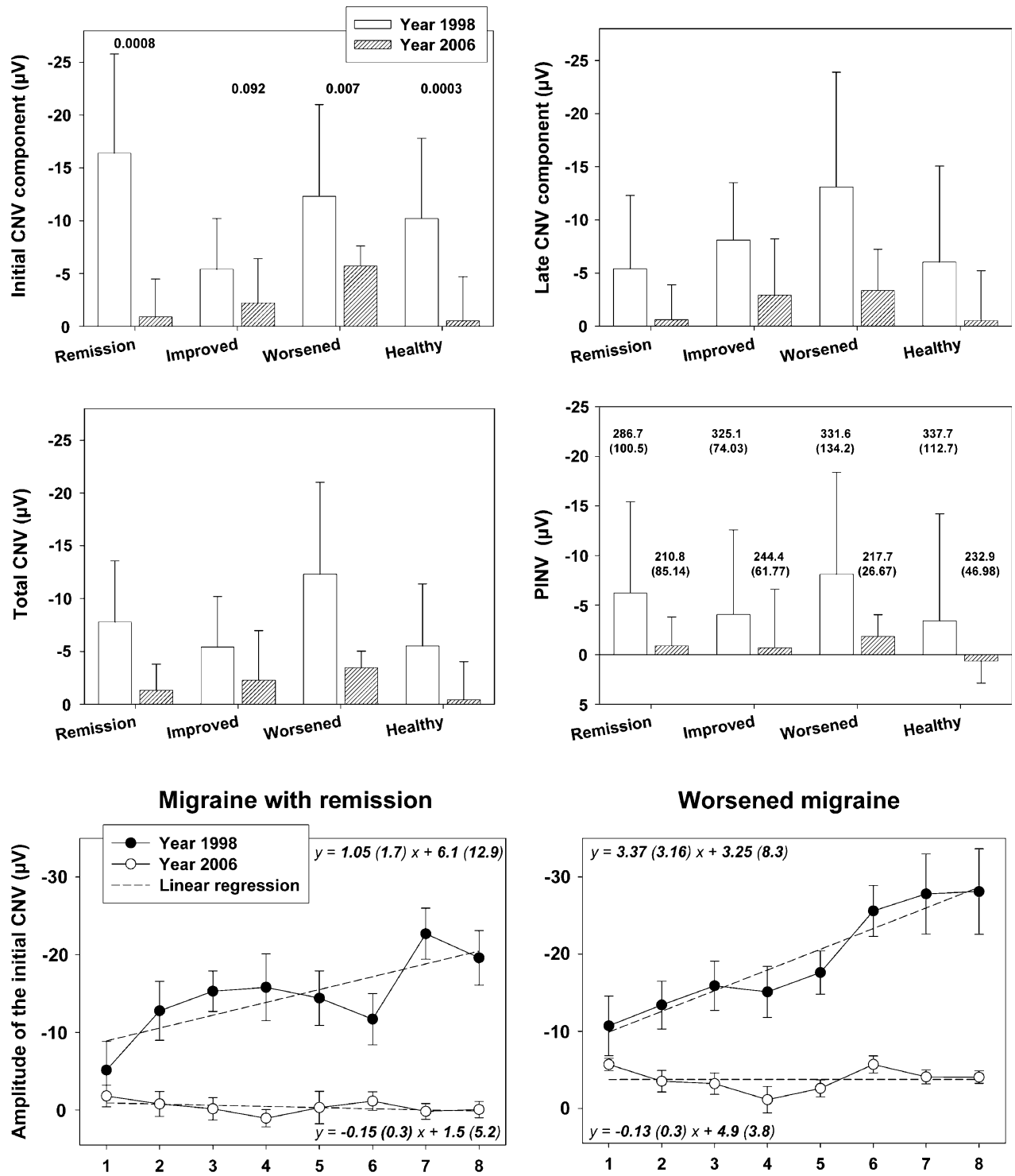

Worsened migraine

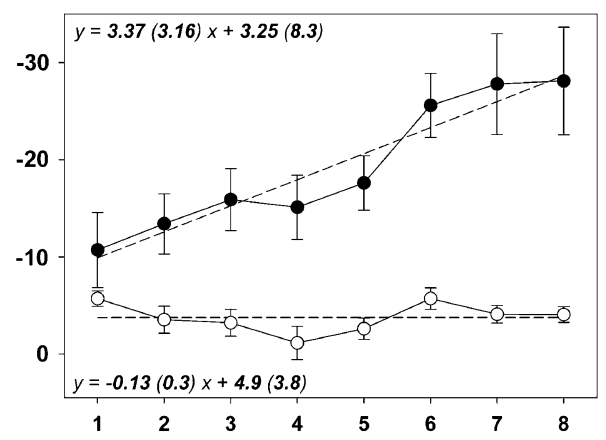

Improved migraine

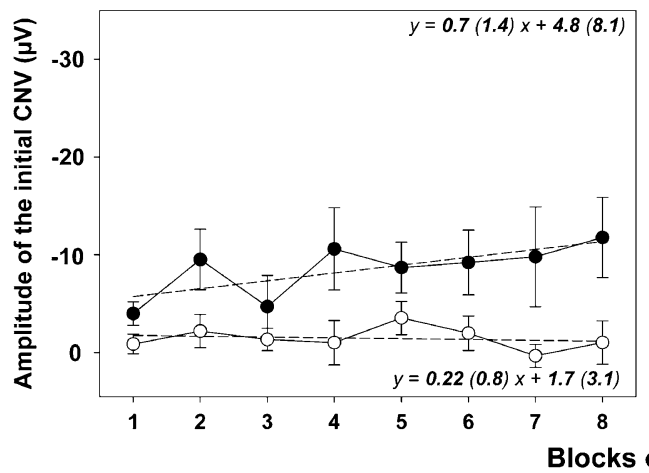

Healthy subjects

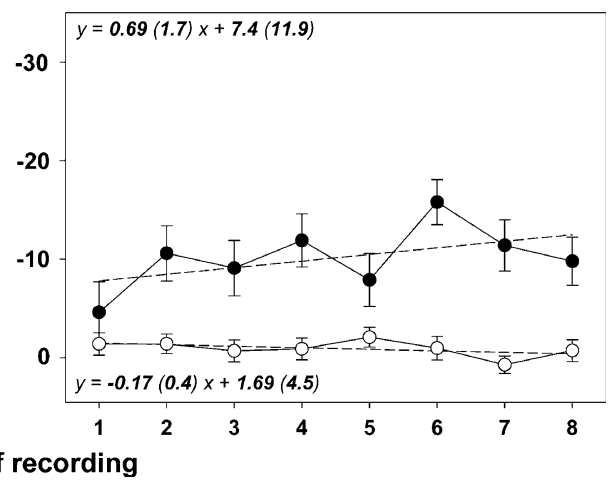

were in remission at $\mathrm{T} 2$ and in migraine patients with worsening of headaches compared with migraineurs with improved headaches (corrected $P=0.034$ and $P=0.046$, respectively). At $\mathrm{T} 2$, only patients with worsening of headaches were characterized by significantly higher iCNV amplitudes compared with migraineurs who were in remission at $\mathrm{T} 2(P=0.01$, corrected $)$ and healthy subjects $(P=0.04$, corrected $)$.

Migraine patients who were in remission at T2 and healthy subjects demonstrated the most pronounced 
Table 2 Results of ANOVA with the within-subjects factor Follow-up, between-subjects factor Group and interaction Follow-up $\times$ Group for amplitudes of all CNV components, regression coefficient characterizing iCNV habituation (Hab.A) and reaction time (RT)

\begin{tabular}{lllllll}
\hline & Total CNV & Initial CNV & Late CNV & PINV & Hab. A & RT \\
\hline Follow-up & $\mathbf{2 4 . 8} /<\mathbf{0 . 0 0 1}$ & $\mathbf{5 7 . 2 / < 0 . 0 0 1}$ & $\mathbf{1 3 . 2} /<\mathbf{0 . 0 0 1}$ & $\mathbf{5 . 3 / 0 . 0 2 6}$ & $\mathbf{1 9 . 9 / < 0 . 0 0 1}$ \\
Group & $2.12 / 0.102$ & $\mathbf{5 . 3 6 / 0 . 0 0 3}$ & $2.37 / 0.083$ & $0.64 / 0.610$ & $\mathbf{3 . 5 1 / 0 . 0 2 3}$ & $1.32 / 0.280$ \\
Follow-up $\times$ Group & $0.99 / 0.402$ & $\mathbf{3 . 4 7 / 0 . 0 2 4}$ & $0.31 / 0.825$ & $0.09 / 0.965$ & $\mathbf{3 . 0 4 / 0 . 0 3 9}$ & $1.27 / 0.296$ \\
\hline
\end{tabular}

Results are given as $F$ value/ $P$ value. Degrees of freedom for Follow-up: 1, 44; for Group and Follow-up $\times$ Group: 3, 44. Significant results are shown in bold

reduction of the iCNV amplitude in the 8-year follow-up. Two-tailed paired-samples $t$ tests for comparisons between iCNV amplitudes at $\mathrm{T} 1$ and $\mathrm{T} 2$ within each group (see Fig. 1 for the iCNV component) show that the most clear reduction of the iCNV over time was observed in healthy subjects and migraine patients with remission, less pronounced reduction was seen in the migraine group with worsening of headaches, and the reduction of the iCNV in the group with improved headaches was even non-significant. Concerning coefficient of the iCNV habituation, migraine patients with worsening of headaches demonstrated the most pronounced reduction of the coefficient in the 8-year follow-up. However, not all comparisons of habituation coefficients between $\mathrm{T} 1$ and $\mathrm{T} 2$ with $\mathrm{t}$ tests survived Bonferroni alpha adjustment.

\section{Discussion}

This study provides the following main findings: (1) 33\% of migraine patients experienced spontaneous remission in the 8 years follow-up; (2) amplitudes of all CNV components decreased and habituation of the iCNV increased in the observed time; (3) the reduction of the iCNV amplitude was more pronounced in migraine patients who were in remission at $\mathrm{T} 2$ and in healthy subjects and less pronounced in both migraine groups with persisting headaches; (4) patients with worsened headache demonstrated the most pronounced loss of iCNV habituation at T1; (5) the iCNV amplitude at T2 differentiates patients with worsened migraine (larger iCNV amplitude) from patients with remission or healthy control subjects.

Prognosis of migraine in childhood and adolescents

Prospective studies have shown that migraine remits in 17$48 \%$ of subjects, persists in $20-81 \%$ of patients, and transforms into other types of headache in $11-37 \%$ of cases $[29,30]$. In patients with persisting migraine, the migrainous headache improved in the majority of them [31, $32]$. Our data (33\% remission and $44 \%$ improvement) are well in line with previous studies. However, we did not find transformation of migraine into other types of primary headaches, unlike other studies. This discrepancy may be explained by certain methodological features. It is important to emphasize that the aim of our study in the year 1998 was to investigate the relationship between clinical course of migraine, psychiatric comorbidity and neurophysiological parameters in migraine families [13, 33]. The study was conceptualized as a neurophysiological study rather than an epidemiological one. For that purpose, only patients with a strong diagnosis of migraine without aura were recruited: all patients were required to use a daily headache diary to report and document in vomiting during migraine attacks, severe headaches accompanied by pronounced disability or positive family history in addition to the fulfillment of the IHS criteria for migraine. Recruitment was carried out from a clinical sample of patients which had not participated in any prior treatment programs. In the course of the 8-year follow-up, only $18 \%$ were treated mostly with behavioral therapy in years 1998-2000. Therefore, the clinical course of migraine was spontaneous in the majority of our patients. Moreover, the remission, improvement and worsening of migraine in our study were determined based on information from both clinical interviews and headache diaries which compared the clinical course of migraine in the years 1998 and 2006. Most previous studies used only interviews or questionnaires to assess the clinical course. Indeed, there is evidence that patients underestimate the frequency and accompanying symptoms and overestimate the duration and severity of migraine attacks in interviews compared with diaries [34, 35]. In our opinion, the sample of participants in our study is insufficient to be able to address epidemiological questions about the prognosis of migraine. However, this sample does have advantages for use in neurophysiological investigations.

\section{$\mathrm{CNV}$ changes over time}

In this longitudinal study, all components of the CNV significantly decreased and the habituation of the iCNV increased during the observed 8 years. These results correspond with our previous cross-sectional study which 
demonstrated a pronounced reduction in the iCNV amplitude and an increase in iCNV habituation with age in healthy subjects in late adolescence and early adulthood [17]. Indeed, healthy pre-school and primary school children are characterized by significantly larger iCNV amplitudes and more pronounced loss of the iCNV habituation than healthy adults $[13,17]$. These developmental CNV features of children were interpreted in terms of the influence of cerebral maturation on processes of cortical excitability and information processing in healthy individuals $[8,13,17,22,23]$. Note that the longitudinal study demonstrated all $\mathrm{CNV}$ components undergoing changes and reduction over 8 years. It seems likely that the overall voltage of cortical negativity over the vertex decreases with time. Since all components are influenced by maturation, the results are difficult to interpret in terms of the functional significance of each particular component. It may be misleading to say that the reduction of iCNV and the increase in its habituation with time may reflect the maturation of orienting reactions and early motor preparation as part of response anticipation $[5,6,13,36]$, reduction of the $1 \mathrm{CNV}$ may be attributed to the maturation of advanced motor preparation and sensory attention [7, 8], and the reduction of PINV may represent the maturation of movement and contingency evaluation [37, 38]. It is more likely that developmental changes influenced unspecific mechanisms which underly all components, such as mechanisms which regulate cortical excitability [4]. Taking into account the recent work of Bender et al. [23], it may be suggested that the reduction of the $\mathrm{CNV}$ voltage over $\mathrm{Cz}$ with increasing age may be due to developmental changes in modulatory circuits of the brainstem, i.e. due to reduced influences of the unspecific ascending reticular formation on the excitability in the cortico-striato-thalamocortical loop [3, 39]. Alternatively, our results may be explained by increasing influence of prefrontal cortex on thalamo-cortical circuitry, since the control function of the prefrontal cortex matures into late adolescence/early adulthood [40]. Finally, the described neurophysiological changes may be related to an unspecific decrease of overall cortical excitability with age. Different studies have shown that the amplitudes of negative evoked potentials decrease and positive potentials increase with increasing age [41, 42]. Event-related potential negativity is interpreted as a representation of cortical excitability, facilitating the processing of sensory input, while positivity is a manifestation of neuronal inhibition $[3,4]$. Therefore, sensory maturation results in the predominance of inhibitory over facilitatory processing seen in reduction of all negative components of evoked potentials, including the CNV. Our data cannot pinpoint which particular mechanism underlies CNV changes with increasing age. One possible way to answer this question is the analysis of age-related changes in $\mathrm{CNV}$ topography [8, 23, 43]. However, the data from 1998 were obtained only from $\mathrm{Cz}$, so investigation of topography is not possible in this study.

\section{Differences between migraineurs and healthy subjects}

Both migraine patients who were in remission in 2006 and healthy subjects demonstrated equal trajectories of cerebral maturation. However, migraine patients with persistence of headaches were characterized by a less pronounced reduction of the iCNV amplitude in the 8-year follow-up. This longitudinal study supports previous observations from our cross-sectional study which showed that migraineurs were characterized by a delay in cerebral maturation [17]. It seems likely that the persistence of headaches is an important factor influencing cerebral maturation of the iCNV. Indeed, most patients in the study of Kropp et al. [17] suffered substantially under headaches. The burden from migraine and active manifestation of the disease may explain differences in iCNV amplitude between healthy subjects and migraine patients observed in previous studies [5, 10-17, 27, 33]. This aspect is supported by this study. Patients with worsened migraine differed significantly from migraineurs who demonstrated remission or healthy subjects at $\mathrm{T} 2$ for the amplitude of the iCNV. It seems likely that the genetic effect on $\mathrm{CNV}$ in migraine is compromised by the described effect of brain maturation. Previous studies have demonstrated a genetic contribution to the variance of the $\mathrm{iCNV}$ amplitude and habituation in migraine [13, 44]. However, this contribution may be shown only for patients and their relatives (adolescents and young adults) which finished maturation of brain functions associated with CNV [44]. Therefore, age of patients and developmental stage of their brain functions have to be taken into account by investigation of genetic effects on brain activity in migraine.

The influence of migraine manifestation on $\mathrm{CNNV}$ and its maturation was not described for the coefficient of habituation. Although the reduction of the coefficient in the 8 -year follow-up was more pronounced in the group with worsened migraine, this effect may be attributed to a significantly more pronounced loss of the iCNV habituation in this group in 1998. In 2006, all groups of migraine patients and healthy subjects demonstrated equal iCNV habituation. This finding is in contrast with the study of Kropp et al. [17] which has demonstrated a clear reduction of the habituation coefficient with age in healthy subjects but not in migraine patients. There are different explanation for the described differences between the study of Kropp et al. [17] and our data. (1) At T1 and T2, we investigated patients with a spontaneous clinical course of migraine who were recruited from the database of the University Hospital of Paediatric Neurology. Most children had been 
referred to the clinic for diagnosis only (only $18 \%$ were motivated to be treated). It seems likely that migraine only had a limited impact on quality of life in the majority of our patients. The patients in the study of Kropp et al. [17] had been referred to the Institute of Medical Psychology between 1990 and 1998 for treatment. The recruitment bias in the cross-sectional study of Kropp et al. [17] may have misled the course of maturation of the iCNV habituation. This bias is resolved by the employment of a longitudinal design. (2) Although 8 years passed from the first recording, the recording at T2 was the repetition. In 1998, the children were younger and may have reacted more sensitively to the novel situation associated with neurophysiological recording. The impact of this at T1 may have led to reduced CNV habituation, which subsequently normalized at T2 in most of the subjects investigated, irrespective of diagnosis. Moreover, a statistical regression to the mean in all groups cannot be ruled out, because the iCNV habituation in children was minimal at $\mathrm{T} 1$.

This study provided an additional interesting finding: patients with worsened migraine at $\mathrm{T} 2$ demonstrated a pronounced loss of iCNV habituation at T1. It seems likely that the iCNV habituation is associated with a poor clinical prognosis of migraine. It is difficult, however, to estimate the significance of this finding because of a small number of patients in the group with worsened migraine. To prove the validity of these observation and to investigate the significance of other neurophysiological parameters for the prognosis of migraine further large-scale longitudinal studies are needed. Moreover, larger group of newly diagnosed subjects with migraine is needed to make conclusions about the value of $\mathrm{CNV}$ as a predictor for development of migraine over time.

Conflict of interest None.

\section{References}

1. Schoenen J (2006) Neurophysiological features of the migraineous brain. Neurol Sci 27(Suppl 2):77-81

2. Schoenen J, Ambrosini A, Sandor PS, Maertens de Noordhout A (2003) Evoked potentials and transcranial magnetic stimulation in migraine: published data and viewpoint on their pathophysiological significance. Clin Neurophysiol 114:955-972

3. Birbaumer N, Elbert T, Canavan A, Rockstroh B (1990) Slow potentials of the cerebral cortex and behaviour. Physiol Rev 70:1-41

4. Elbert T (1993) Slow cortical potentials reflect the regulation of cortical excitability. In: McCallum WC, Curry SH (eds) Slow potentials changes in the human brain. Plenum Press, New York, pp 235-251

5. Siniatchkin M, Kropp P, Gerber WD, Voznesenskaya T, Vein AM (2000) Are the periodic changes of neurophysiological parameters during the pain-free interval in migraine related to abnormal orienting activity? Cephalalgia 20:20-29
6. Bender S, Resch F, Weisbrod M, Oelkers-Ax R (2004) Specific task anticipation versus unspecific orienting reaction during early contingent negative variation. Clin Neurophysiol 115:1836-1845

7. Cui RQ, Egkher A, Huter D, Lang W, Lindinger G, Deeke L (2000) High resolution spatiotemporal analysis of the contingent negative variation in simple or complex motor tasks and nonmotor task. Clin Neurophysiol 111:1847-1859

8. Bender S, Weisbrod M, Bornfleth H, Resch F, Oelkers-Ax R (2005) How do children prepare to react? Imaging maturation of motor preparation and stimulus anticipation by late contingent negative variation. NeuroImage 27:737-752

9. Maertens de Noordhout A, Timsit-Bertheir M, Timsit M, Schoenen J (1986) Contingent negative variation in headache. Ann Neurol 19:78-80

10. Kropp P, Gerber WD (1993) Is increased amplitude of contingent negative variation in migraine due to cortical hyperactivity or to reduced habituation? Cephalalgia 13:37-41

11. Kropp P, Gerber WD (1998) Prediction of migraine attacks using a slow cortical potential, the contingent negative variation. Neurosci Lett 257:73-76

12. Siniatchkin M, Gerber WD, Kropp P, Vein A (1999) How the brain anticipates an attack - a study of neurophysiological periodicity in migraine. Funct Neurol 14:69-78

13. Siniatchkin M, Kirsch E, Kropp P, Gerber WD, Stephani U (2000) Slow cortical potentials in migraine families. Cephalalgia 20:881-892

14. Siniatchkin M, Kropp P, Gerber WD (2000) Migraine in childhood: are periodically occurring migraine attacks related to dynamic changes of cortical information processing? Neurosci Lett 279:1-4

15. Siniatchkin M, Averkina N, Gerber WD (2006) Relationship between precipitating agents and neurophysiological abnormalities in migraine. Cephalalgia 26:457-465

16. Siniatchkin M, Averkina N, Andrasik F, Stephani U, Gerber WD (2006) Neurophysiological reactivity before a migraine attack. Neurosci Lett 400:121-124

17. Kropp P, Siniatchkin M, Stephani U, Gerber WD (1999) Migraine-evidence for a disturbance of cerebral maturation in man? Neurosci Lett 276:181-184

18. Siniatchkin M, Kropp P, Neumann M, Gerber WD, Stephani U (2000) Intensity dependence of auditory evoked cortical potentials in migraine families. Pain 85:247-254

19. Siniatchkin M, Kropp P, Stephani U, Gerber WD (2000) Maturation of sensory information processing: evidence from the intensity dependence of AEP. J Psychophysiol 14:59

20. Oelkers-Ax R, Bender S, Just U, Pfuller U, Parzer P, Resch F et al (2004) Pattern-reversal visual-evoked potentials in children with migraine and other primary headache: evidence for maturation disorder? Pain 108:267-275

21. Oelkers-Ax R, Parzer P, Resch F, Weisbrod M (2005) Maturation of early visual processing investigated by a pattern-reversal habituation paradigm is altered in migraine. Cephalalgia 25:280289

22. Bender S, Weisbrod M, Just U, Pfuller U, Parzer P, Resch F et al (2002) Lack of age-dependent development of the contingent negative variation $(\mathrm{CNV})$ in migraine children? Cephalalgia 22:132-136

23. Bender S, Weisbrod M, Resch F, Oelkers-Ax R (2007) Stereotyped topography of different elevated contingent negative variation components in children with migraine without aura points towards a subcortical dysfunction. Pain 127:221-233

24. Oelker-Ax R, Schmidt K, Bender S, Reimer I, Möhler E, Knauss E, Resch F, Weisbrod M (2008) Longitudinal assessment of response preparation and evaluation in migraine gives evidence for deviant maturation. Cephalalgia 28:237-249 
25. IHS (2004) The International Classification of Headache Disorders, 2nd edn, vol 24. Headache Classification Committee of the International Headache Society. Cephalalgia, pp 1-160

26. Pothmann R (1993) Migräne im Kindesalter. Erläuterungen zum „Migränetagebuch für Kinder” für die Handhabung in der Praxis. Arcis Verlag, München

27. Böcker KB, Timsit-Berthier M, Schoenen J, Brunia CH (1990) Contingent negative variation in migraine. Headache 30:604-609

28. Petrinovich L, Widaman KF (1984) An evaluation of statistical strategies to analyse repeated-measures data. In: Peeke HVS, Petrinovich L (eds) Habituation, sensitization and behaviour. Academic Press, New York, pp 156-204

29. Congdon PJ, Forsythe WI (1979) Migraine in childhood. A review. Clin Pediatr 18:353-359

30. Monastero R, Camarda C, Pipia C, Camarda R (2006) Prognosis of migraine headaches in adolescents: a 10-year follow-up study. Neurology 67:1353-1356

31. Dooley J, Bagnell A (1995) The prognosis and treatment of headaches in children-a ten year follow-up. Can J Neurol Sci 22:47-49

32. Guidetti V, Galli F (1998) Evolution of headache in childhood and adolescence: an 8-year follow-up. Cephalalgia 18:449-454

33. Gerber WD, Stephani U, Kirsch E, Kropp P, Siniatchkin M (2001) Slow cortical potentials in migraine families are associated with psychosocial factors. J Psychosom Res 52:215-222

34. Metsähonkala L, Sillanpää M, Tuominen J (1997) Headache diary in the diagnosis of childhood migraine. Headache 37:240 244

35. Van den Brink M, Bandel-Hoekstra EM, Abu-Saad H (2001) The occurrence of recall bias in paediatric headache: comparison of questionnaire and diary data. Headache 41:11-20
36. Gomez CM, Marco J, Grau C (2003) Preparatory visuo-motor cortical network of the contingent negative variation estimated by current density. NeuroImage 20:216-224

37. Klein C, Rockstroh B, Cohen R, Berg P (1996) Contingent negative variation (CNV) and determinant of the post-imperative negative variation (PINV) in schizophrenic patients and healthy controls. Schizophr Res 21:97-110

38. Verleger R, Wascher E, Arolt V, Daase C, Strohm A, Kompf D (1999) Slow EEG potentials (contingent negative variation and post-imperative negative variation) in schizophrenia: their association to the present state and to Parkinsonian medication effects. Clin Neurophysiol 110:1175-1192

39. Mayes LC (2000) A developmental perspective on the regulation of arousal states. Semin Perinatol 24:267-279

40. Segalowitz SJ, Davies PL (2004) Charting the maturation of the frontal lobe: an electrophysiological strategy. Brain Cogn 55:116-133

41. Polich J, Ladish C, Burns T (1990) Normal variation of P300 in children: age, memory span, and head size. Int J Psychophysiol 9:237-248

42. Kok A (1999) Varieties of inhibition: manifestation in cognition, event-related potentials and aging. Acta Psychol (Amst) 101:129-158

43. Bender S, Oelkers-Ax R, Resch F, Weisbrod M (2006) Frontal lobe involvement in the processing of meaningful auditory stimuli develops during childhood and adolescence. NeuroImage 33:759-773

44. Siniatchkin M, Kropp P, Gerber WD (2001) Contingent negative variation in subjects at risk for migraine without aura. Pain 94:159-167 